\title{
Effectiveness of short-term air quality emission controls: a high-resolution model study of Beijing during the Asia-Pacific Economic Cooperation (APEC) summit period
}

Tabish Umar Ansari et al.

Correspondence to: Tabish Umar Ansari (t.ansari@lancaster.ac.uk) and Oliver Wild (o.wild@lancaster.ac.uk)

The copyright of individual parts of the supplement might differ from the CC BY 4.0 License. 
Table S1. Comparison of observed and simulated meteorological variables for the entire period using FNL and ECMWF fields

\begin{tabular}{|c|c|c|c|c|c|c|c|c|c|c|}
\hline & \multirow{2}{*}{$\begin{array}{c}\text { Number of } \\
\text { Stations }\end{array}$} & \multirow[t]{2}{*}{ Obs. avg. } & \multicolumn{2}{|c|}{ Sim avg } & \multicolumn{2}{|c|}{ Bias } & \multicolumn{2}{|c|}{ RMSE } & \multicolumn{2}{|r|}{$\mathrm{r}$} \\
\hline & & & FNL & ECMWF & FNL & ECMWF & FNL & ECMWF & FNL & ECMWF \\
\hline \multicolumn{11}{|c|}{ 2-m Temperature $\left({ }^{\circ} \mathrm{C}\right)$} \\
\hline Beijing & 1 & 9.68 & 11.52 & 11.44 & 1.84 & 1.76 & 3.28 & 3.36 & 0.88 & 0.87 \\
\hline D03 & 30 & 8.91 & 8.98 & 8.95 & 0.07 & 0.04 & 2.47 & 2.46 & 0.94 & 0.94 \\
\hline D02 & 77 & 7.87 & 7.53 & 7.55 & -0.34 & -0.32 & 2.39 & 2.35 & 0.95 & 0.95 \\
\hline D01 & 324 & 9.62 & 7.77 & 7.79 & -1.85 & -1.83 & 3.23 & 3.23 & 0.94 & 0.94 \\
\hline \multicolumn{11}{|c|}{ 2-m Relative Humidity (\%) } \\
\hline Beijing & 1 & 54.7 & 34.1 & 39.1 & -20.6 & -15.6 & 26.9 & 22.4 & 0.77 & 0.81 \\
\hline D03 & 30 & 54.9 & 44.8 & 48.9 & -10.1 & -6.0 & 19.6 & 16.7 & 0.75 & 0.78 \\
\hline D02 & 77 & 54.4 & 47.8 & 51.1 & -6.6 & -3.3 & 17.4 & 15.2 & 0.74 & 0.78 \\
\hline D01 & 324 & 62.8 & 60.4 & 62.6 & -2.4 & -0.2 & 16.8 & 15.6 & 0.73 & 0.76 \\
\hline \multicolumn{11}{|c|}{ 10-m Wind Speed $\left(\mathrm{m} \mathrm{s}^{-1}\right)$} \\
\hline Beijing & 1 & 5.41 & 2.27 & 2.24 & -3.14 & -3.17 & 4.98 & 5.09 & 0.72 & 0.69 \\
\hline D03 & 30 & 5.73 & 3.26 & 3.20 & -2.47 & -2.53 & 4.60 & 4.65 & 0.62 & 0.61 \\
\hline D02 & 77 & 6.18 & 3.60 & 3.55 & -2.58 & -2.63 & 4.52 & 4.55 & 0.67 & 0.66 \\
\hline D01 & 324 & 5.67 & 3.38 & 3.36 & -2.29 & -2.31 & 4.29 & 4.30 & 0.60 & 0.61 \\
\hline \multicolumn{11}{|c|}{ 10-m Wind Direction $\left(^{\circ}\right)$} \\
\hline Beijing & 1 & 197.5 & 214.2 & 191.0 & 16.7 & -6.6 & 73.9 & 73.9 & 0.79 & 0.80 \\
\hline D03 & 30 & 215.1 & 210.0 & 206.5 & -6.9 & -8.6 & 62.7 & 63.4 & 0.78 & 0.78 \\
\hline D02 & 77 & 214.4 & 212.2 & 208.9 & -2.8 & -5.5 & 65.4 & 65.4 & 0.76 & 0.76 \\
\hline D01 & 324 & 206.5 & 193.4 & 188.4 & -13.1 & -18.0 & 71.9 & 72.2 & 0.74 & 0.74 \\
\hline
\end{tabular}

Hourly values are used for each station from 12 October to 19 November 2014 . Where observation data are missing, model values were removed to ensure that sampling was consistent. 
Table S2. Meteorological performance over Beijing during pre-APEC and APEC period

\begin{tabular}{|c|c|c|c|c|c|}
\hline & Obs. avg. & Sim. avg. & Mean bias & RMSE & $\mathrm{R}$ \\
\hline \multicolumn{6}{|c|}{$2 \mathrm{~m}$ Temperature $\left({ }^{\circ} \mathrm{C}\right)$} \\
\hline Episode 1 & 14.63 & 16.84 & 2.21 & 3.79 & 0.79 \\
\hline Episode 2 & 12.15 & 14.47 & 2.32 & 3.28 & 0.74 \\
\hline Episode 3 & 11.35 & 11.76 & 0.41 & 1.78 & 0.86 \\
\hline APEC period & 7.14 & 9.14 & 2.00 & 3.76 & 0.82 \\
\hline \multicolumn{6}{|c|}{ Relative Humidity (\%) } \\
\hline Episode 1 & 61.9 & 41.4 & -20.6 & 26.6 & 0.76 \\
\hline Episode 2 & 74.3 & 52.7 & -21.5 & 26.0 & 0.74 \\
\hline Episode 3 & 55.6 & 41.3 & -14.3 & 19.5 & 0.89 \\
\hline APEC period & 47.0 & 34.4 & -12.6 & 21.2 & 0.82 \\
\hline \multicolumn{6}{|c|}{ Wind Speed $\left(\mathrm{m} \mathrm{s}^{-1}\right)$} \\
\hline Episode 1 & 5.30 & 2.45 & -2.85 & 4.86 & 0.74 \\
\hline Episode 2 & 3.50 & 1.82 & -1.68 & 2.76 & 0.56 \\
\hline Episode 3 & 5.46 & 2.07 & -3.39 & 5.35 & 0.79 \\
\hline APEC period & 6.50 & 2.56 & -3.94 & 6.06 & 0.67 \\
\hline \multicolumn{6}{|c|}{ Wind Direction $\left(^{\circ}\right)$} \\
\hline Episode 1 & 176.9 & 172.9 & -4.1 & 63.5 & 0.78 \\
\hline Episode 2 & 148.6 & 141.4 & -7.3 & 74.9 & 0.85 \\
\hline Episode 3 & 143.6 & 163.4 & 19.7 & 62.1 & 0.88 \\
\hline APEC period & 257.3 & 220.8 & -36.5 & 69.4 & 0.79 \\
\hline
\end{tabular}

Episode 1: 15-20 Oct, Episode 2: 21-25 Oct, Episode 3: 26 Oct-1 Nov, APEC period: 3-12 Nov 
Table S3. Comparison of pollutant concentrations with network measurements over the period 12-31 October 2014

\begin{tabular}{|c|c|c|c|c|c|c|c|}
\hline & $\begin{array}{c}\text { Number of } \\
\text { Stations }\end{array}$ & Obs & Sim & Bias & $\begin{array}{c}\text { RMSE } \\
\text { hourly/daily }\end{array}$ & $\begin{array}{c}\mathrm{r} \\
\text { hourly/daily }\end{array}$ & $\begin{array}{c}\text { slope } \\
\text { hourly/daily }\end{array}$ \\
\hline \multicolumn{8}{|l|}{$\mathrm{PM}_{2.5}\left(\mu \mathrm{g} \mathrm{m}^{-3}\right)$} \\
\hline Beijing stations & 12 & 108.3 & 126.2 & 17.9 & $86.7 / 66.7$ & $0.68 / 0.78$ & $0.83 / 0.93$ \\
\hline D03 & 137 & 92.6 & 109.3 & 16.7 & $72.2 / 52.2$ & $0.63 / 0.74$ & $0.71 / 0.80$ \\
\hline D02 & 375 & 75.8 & 87.9 & 12.1 & $63.9 / 48.6$ & $0.60 / 0.69$ & $0.65 / 0.71$ \\
\hline D01 & 1312 & 71.1 & 74.8 & 3.7 & $61.1 / 50.2$ & $0.47 / 0.53$ & $0.48 / 0.54$ \\
\hline \multicolumn{8}{|l|}{$\mathrm{PM}_{10}\left(\mu \mathrm{g} \mathrm{m}^{-3}\right)$} \\
\hline Beijing stations & 12 & 155.4 & 141.5 & -13.9 & $96.5 / 74.0$ & $0.65 / 0.77$ & $0.79 / 0.98$ \\
\hline D03 & 137 & 165.7 & 122.9 & -42.8 & $104.0 / 82.1$ & $0.57 / 0.68$ & $0.50 / 0.58$ \\
\hline D02 & 375 & 138.0 & 98.6 & -39.4 & $94.3 / 75.8$ & $0.54 / 0.65$ & $0.44 / 0.52$ \\
\hline D01 & 1312 & 121.0 & 82.2 & -38.8 & $89.0 / 76.7$ & $0.42 / 0.47$ & $0.32 / 0.37$ \\
\hline \multicolumn{8}{|l|}{$\mathrm{CO}(\mathrm{ppm})$} \\
\hline Beijing stations & 12 & 1.11 & 0.94 & -0.17 & $0.63 / 0.43$ & $0.60 / 0.75$ & $0.46 / 0.61$ \\
\hline D03 & 137 & 1.17 & 0.83 & -0.34 & $0.87 / 0.72$ & $0.29 / 0.34$ & $0.21 / 0.22$ \\
\hline D02 & 375 & 1.14 & 0.66 & -0.48 & $0.88 / 0.79$ & $0.33 / 0.37$ & $0.20 / 0.20$ \\
\hline D01 & 1312 & 1.00 & 0.50 & -0.50 & $0.79 / 0.73$ & $0.32 / 0.34$ & $0.13 / 0.14$ \\
\hline \multicolumn{8}{|l|}{$\mathrm{NO}_{2}(\mathrm{ppb})$} \\
\hline Beijing stations & 12 & 39.09 & 36.09 & -3.00 & $19.33 / 11.10$ & $0.62 / 0.80$ & $0.66 / 0.83$ \\
\hline D03 & 137 & 29.75 & 25.88 & -3.87 & $18.95 / 14.32$ & $0.47 / 0.54$ & $0.45 / 0.51$ \\
\hline D02 & 375 & 24.86 & 19.45 & -5.41 & $16.99 / 13.21$ & $0.49 / 0.55$ & $0.44 / 0.50$ \\
\hline D01 & 1312 & 22.73 & 12.45 & -10.28 & $18.33 / 15.44$ & $0.42 / 0.47$ & $0.30 / 0.36$ \\
\hline \multicolumn{8}{|l|}{$\mathrm{SO}_{2}(\mathrm{ppb})$} \\
\hline Beijing stations & 12 & 3.92 & 12.27 & 8.35 & $11.88 / 10.55$ & $0.27 / 0.52$ & $0.68 / 1.74$ \\
\hline D03 & 137 & 13.28 & 14.47 & 1.19 & $13.66 / 9.66$ & $0.21 / 0.31$ & $0.22 / 0.24$ \\
\hline D02 & 375 & 12.23 & 13.21 & 0.98 & $13.19 / 9.01$ & $0.24 / 0.34$ & $0.26 / 0.28$ \\
\hline D01 & 1312 & 10.27 & 8.93 & -1.34 & $11.17 / 8.54$ & $0.19 / 0.28$ & $0.18 / 0.24$ \\
\hline \multicolumn{8}{|l|}{$\mathrm{O}_{3}(\mathrm{ppb})$} \\
\hline Beijing stations & 12 & 12.53 & 12.19 & -0.34 & $13.92 / 6.49$ & $0.47 / 0.67$ & $0.44 / 0.82$ \\
\hline D03 & 137 & 17.76 & 18.75 & 0.99 & $15.96 / 10.88$ & $0.45 / 0.49$ & $0.43 / 0.50$ \\
\hline D02 & 375 & 21.23 & 23.08 & 1.85 & $17.19 / 12.80$ & $0.42 / 0.43$ & $0.37 / 0.40$ \\
\hline D01 & 1312 & 21.44 & 32.29 & 10.85 & $22.44 / 17.03$ & $0.29 / 0.27$ & $0.27 / 0.25$ \\
\hline
\end{tabular}


Table S4. Impacts of model resolution on simulation of hourly pollutant concentrations in Beijing over 12-31 October 2014

\begin{tabular}{|c|c|c|c|c|c|c|c|}
\hline & $\mathrm{N}$ points & Obs mean & Sim mean & Mean Bias & RMSE & $\mathrm{r}$ & slope \\
\hline \multicolumn{8}{|c|}{$\mathrm{PM}_{2.5}\left(\mu \mathrm{g} \mathrm{m}^{-3}\right)$} \\
\hline D03 (3-km) & 3171 & 108.4 & 126.2 & 17.8 & 86.7 & 0.68 & 0.83 \\
\hline D02 (9-km) & 3171 & 108.4 & 128.7 & 20.3 & 87.4 & 0.69 & 0.85 \\
\hline D01 (27-km) & 3171 & 108.4 & 123.1 & 14.7 & 86.1 & 0.68 & 0.81 \\
\hline D01 (no nest) & 3171 & 108.4 & 99.2 & -9.2 & 83.2 & 0.59 & 0.55 \\
\hline \multicolumn{8}{|c|}{$\mathrm{PM}_{10}\left(\mu \mathrm{g} \mathrm{m}^{-3}\right)$} \\
\hline D03 & 2670 & 155.4 & 141.5 & -13.9 & 96.5 & 0.65 & 0.79 \\
\hline D02 & 2670 & 155.4 & 143.6 & -11.8 & 96.6 & 0.65 & 0.80 \\
\hline D01 & 2670 & 155.4 & 137.9 & -17.5 & 96.6 & 0.65 & 0.79 \\
\hline D01 (no nest) & 2670 & 155.4 & 111.2 & -44.2 & 99.8 & 0.58 & 0.54 \\
\hline \multicolumn{8}{|l|}{$\mathrm{CO}(\mathrm{ppm})$} \\
\hline D03 & 3074 & 1.11 & 0.94 & -0.17 & 0.63 & 0.60 & 0.46 \\
\hline D02 & 3074 & 1.11 & 0.95 & -0.16 & 0.61 & 0.61 & 0.47 \\
\hline D01 & 3074 & 1.11 & 0.88 & -0.23 & 0.61 & 0.64 & 0.44 \\
\hline D01 (no nest) & 3074 & 1.11 & 0.68 & -0.43 & 0.73 & 0.62 & 0.31 \\
\hline \multicolumn{8}{|l|}{$\mathrm{NO}_{2}(\mathrm{ppb})$} \\
\hline D03 & 3080 & 39.09 & 36.09 & -3.00 & 19.33 & 0.62 & 0.66 \\
\hline D02 & 3080 & 39.09 & 35.55 & -3.54 & 19.34 & 0.62 & 0.64 \\
\hline D01 & 3080 & 39.09 & 31.92 & -7.17 & 18.33 & 0.67 & 0.62 \\
\hline D01 (no nest) & 3080 & 39.09 & 21.81 & -17.28 & 24.74 & 0.60 & 0.48 \\
\hline \multicolumn{8}{|l|}{$\mathrm{SO}_{2}(\mathrm{ppb})$} \\
\hline D03 & 3074 & 3.92 & 12.27 & 8.35 & 11.88 & 0.27 & 0.68 \\
\hline D02 & 3074 & 3.92 & 12.15 & 8.23 & 11.64 & 0.27 & 0.66 \\
\hline D01 & 3074 & 3.92 & 10.91 & 6.99 & 9.82 & 0.32 & 0.69 \\
\hline D01 (no nest) & 3074 & 3.92 & 6.47 & 2.55 & 5.57 & 0.29 & 0.40 \\
\hline \multicolumn{8}{|l|}{$\mathrm{O}_{3}(\mathrm{ppb})$} \\
\hline D03 & 3046 & 12.56 & 12.19 & -0.37 & 13.92 & 0.47 & 0.44 \\
\hline D02 & 3046 & 12.56 & 12.71 & 0.15 & 13.94 & 0.47 & 0.43 \\
\hline D01 & 3046 & 12.56 & 14.96 & 2.40 & 13.53 & 0.49 & 0.44 \\
\hline D01 (no nest) & 3046 & 12.56 & 17.59 & 5.03 & 14.08 & 0.51 & 0.45 \\
\hline
\end{tabular}



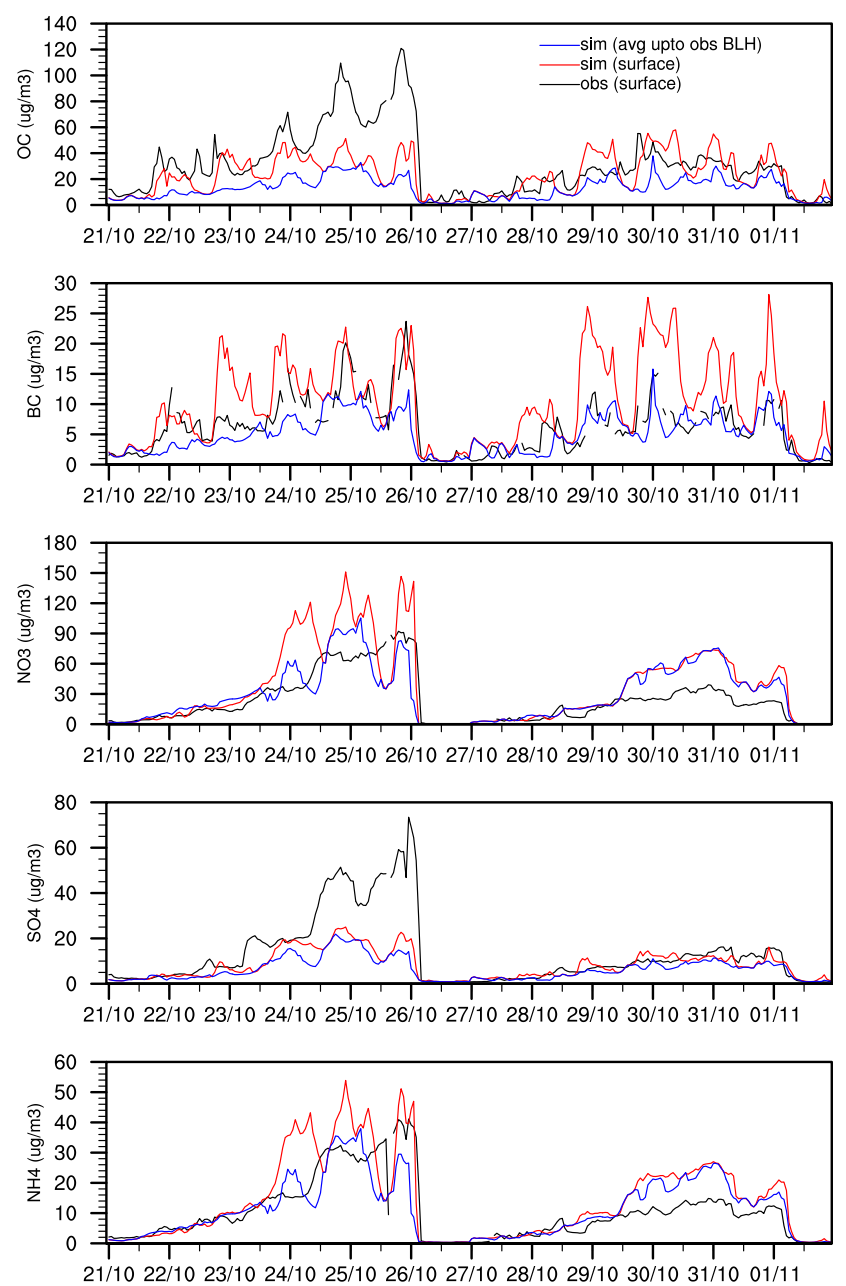

Figure S1. Time-series of simulated aerosol components with and without averaging upto observed boundary layer height against surface observations at the IAP site.

Table S5. Comparison of simulated aerosol components in $\mu \mathrm{g} \mathrm{m}^{-3}$ with and without mixing up to observed boundary layer height agains surface observations at IAP site

\begin{tabular}{lccccccc}
\hline & Obs. avg. & \multicolumn{2}{c}{ Sim avg } & \multicolumn{2}{c}{ Mean Bias } & \multicolumn{2}{c}{ RMSE } \\
Components & & surface & mixed & surface & mixed & surface & mixed \\
\hline OC & 31.08 & 23.24 & 12.86 & -7.84 & -18.22 & 21.43 & 27.30 \\
BC & 6.13 & 10.19 & 4.96 & 4.06 & -1.16 & 5.96 & 3.27 \\
NO3 & 23.30 & 36.73 & 29.42 & 13.44 & 6.12 & 25.47 & 18.00 \\
SO4 & 13.16 & 8.12 & 6.27 & -5.03 & -6.89 & 12.05 & 13.67 \\
NH4 & 10.36 & 14.16 & 11.12 & 3.80 & 0.78 & 8.05 & 6.40 \\
\hline
\end{tabular}


Temperature at IAP tower

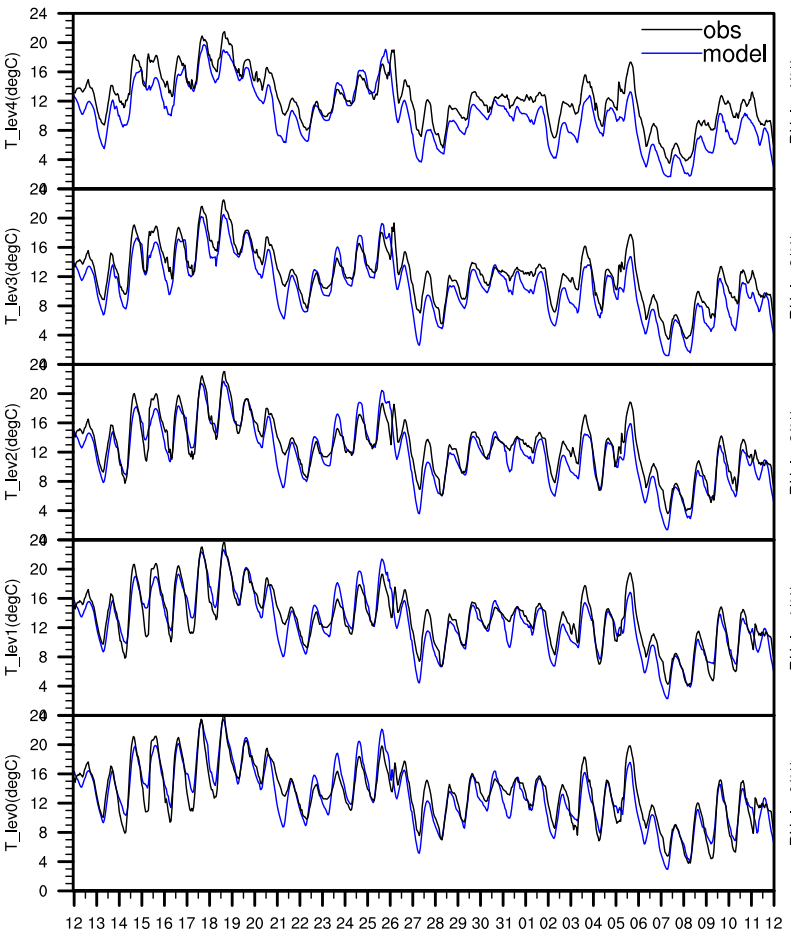

Wind Speed at IAP tower

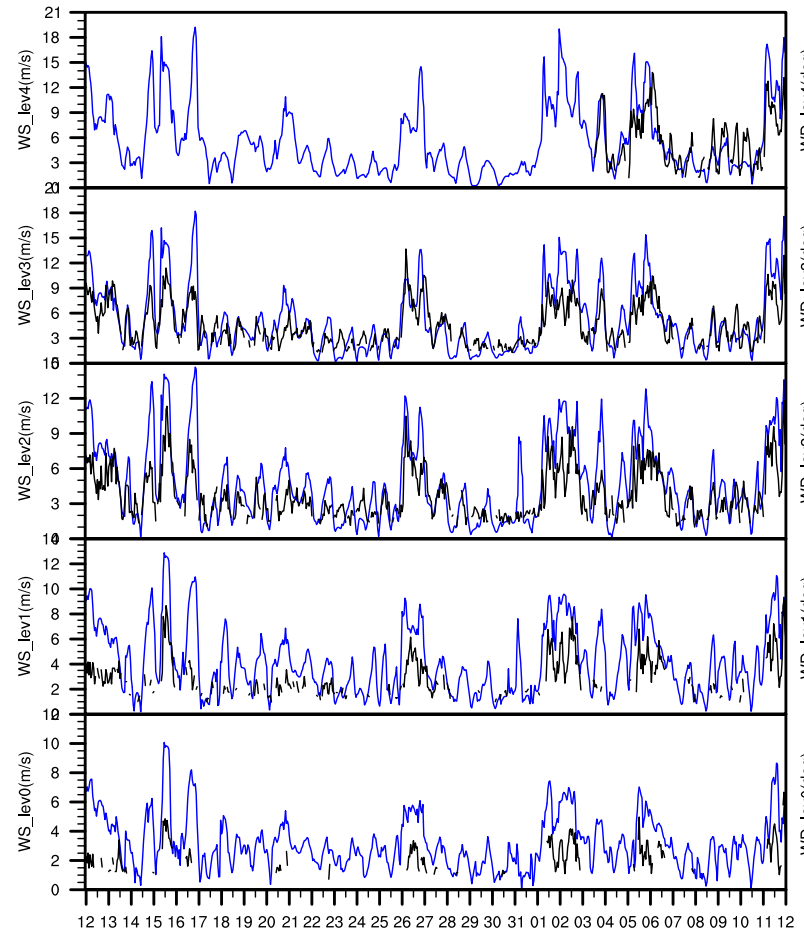

Relative Humidity at IAP tower

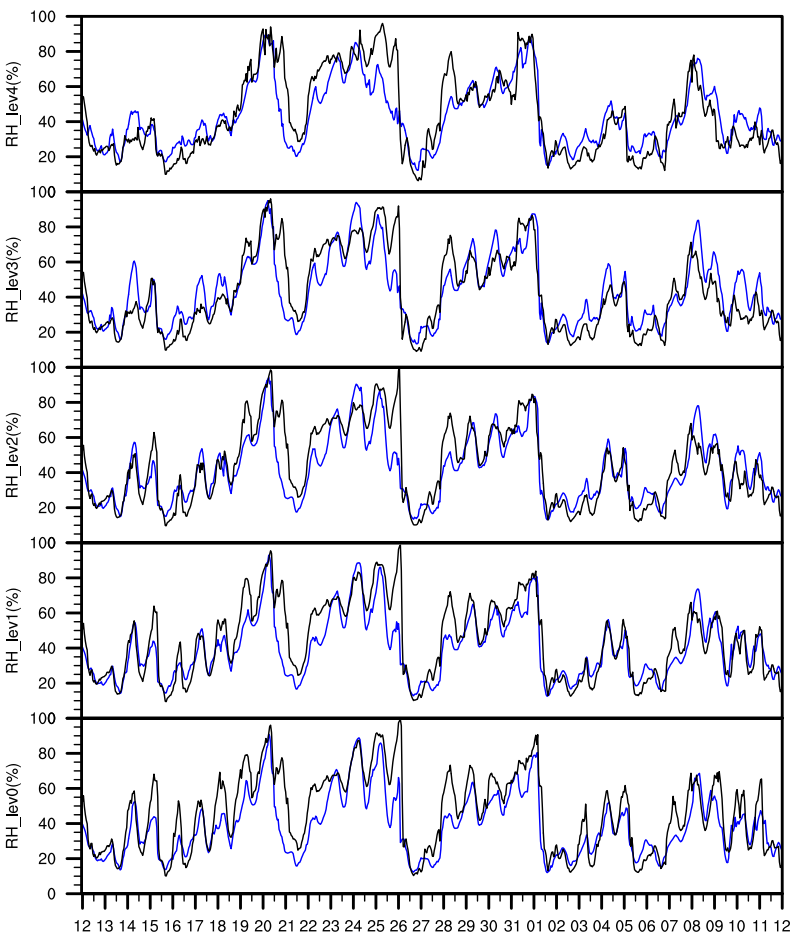

Wind Direction at IAP tower

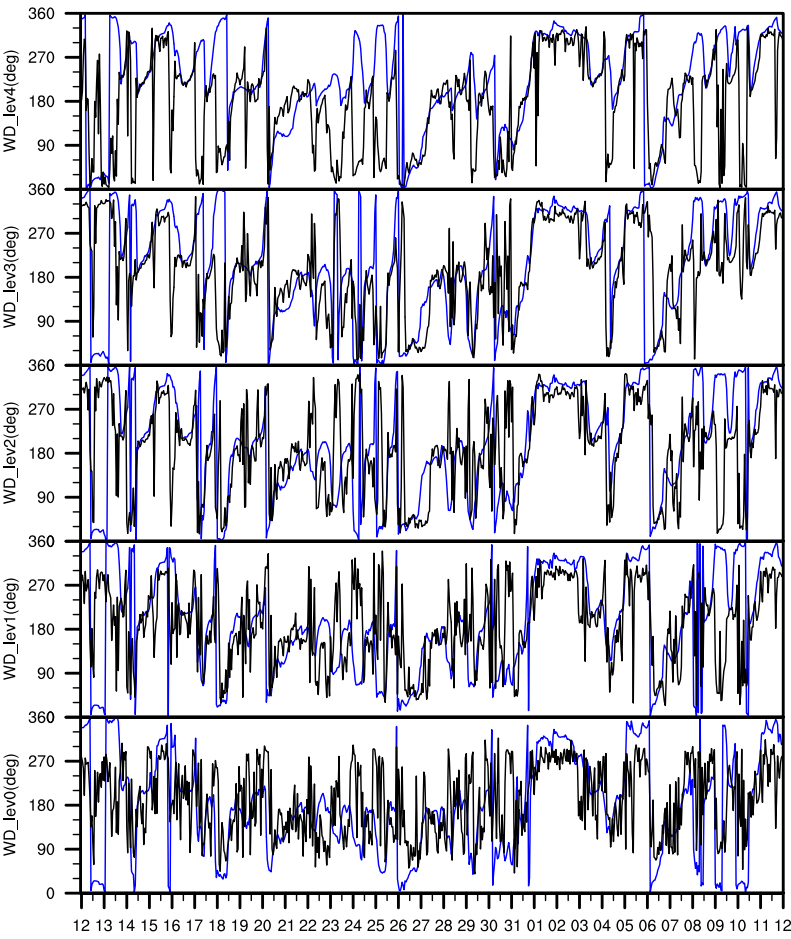

Figure S2. Meteorological evaluation against IAP tower measurements for bottom 5 model levels. lev 0: 0-27 m, lev 1: 27-90 m, lev 2: 90-178 m, lev 3: 178-289 m, lev 4: 289-429 m. 

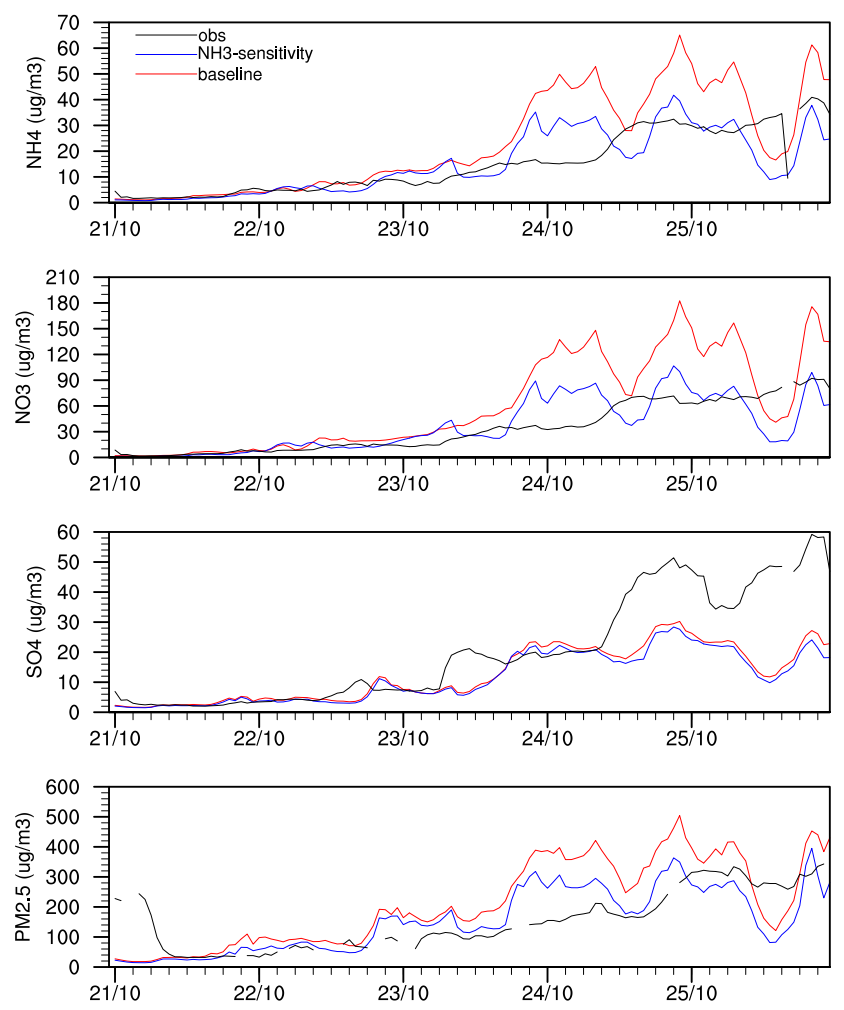

Figure S3. Time-series of aerosol components $\mathrm{NH}_{4}, \mathrm{NO}_{3}$ and $\mathrm{SO}_{4}$ at the IAP site and $\mathrm{PM}_{2.5}$ at Aotizhongxin showing simulated concentrations (in $\mu \mathrm{g} \mathrm{m}^{-3}$ ) from the baseline model run and reduced $\mathrm{NH}_{3}$ emissions run compared to observations. 